\title{
CAMBIOS EN LA ANGULACIÓN DEL PLANO OCLUSAL Y PLANO MANDIBULAR, AL INICIO Y AL FINAL DEL TRATAMIENTO DE ORTODONCIA EN PACIENTES TRATADOS DE LA CLIINICA DE MAESTRÍA EN ORTODONCIA DE LA FACULTAD DE ODONTOLOGÍA DE LA UNIVERSIDAD DE SAN CARLOS DE GUATEMALA DURANTE LOS AÑOS 2014-2018
}

\author{
CHANGES IN THE ANGULATION OF THE OCLUSAL PLANE AND MANDIBULAR \\ PLANE, AT THE BEGINNING AND AT THE END OF ORTHODONTIC TREATMENT IN \\ PATIENTS TREATED AT THE MASTER CLINIC IN ORTHODONTICS OF THE \\ FACULTY OF DENTISTRY OF THE UNIVERSITY OF SAN CARLOS DE GUATEMALA \\ DURING THE YEARS 2014-2018
}

Referencia del artículo

Jofre Ortiz, V. I., Argueta Hernández, B., \& Chinchilla Dubón , G. R. (2021). Cambios en la angulación del plano oclusal y plano mandibular, al inicio y al final del tratamiento de ortodoncia en pacientes tratados de la Clínica de Maestría en Ortodoncia de la Facultad de Odontología de la Universidad de San Carlos de Guatemala dura. Revista Científica del Sistema de Estudios de Postgrado de la Universidad de San Carlos de Guatemala, 4(1), 49-60. DOI: https://doi.org/10.36958/sep.v4i1.76

\author{
Dra. Vilma Isabel Jofre Ortiz \\ vijofre20@gmail.com \\ Cirujana Dentintas \\ Dr. Bienvenido Argueta Hernández \\ bargueta@postgradosodontologia.edu.gt \\ 0000-0001-7121-2009 \\ Doctor en Educación y Estudios Culturales
}

Fecha de recepción: 13/10/2021
Dr. Gerson Rudick Chinchilla Dubón

gchinchilla@postgradosodontologia.edu.gt

id 0000-0001-8538-7969

Cirujano Dentista

Facultad de Odontología

Universidad de San Carlos de Guatemala

Fecha de aceptación: 25/10/2021

\section{RESUMEN}

La inclinación del plano oclusal determina la posición mandibular, tendencia de crecimiento y relación esqueletal. El plano oclusal inclinado, hacia atrás y arriba, corresponde a clase II, en clase III, el ángulo es mas plano, promoviendo movimiento mandibular hacia adelante. El enfoque de tratamiento vertical promueve un movimiento mandibular para corregir los problemas dentoesqueletales. Objetivo: Analizar las diferencias que se manifiestan en el plano oclusal y plano mandibular al inicio y al final del tratamiento de ortodoncia y su correlación, según la relación esqueletal. Método: investigación analítica retrospectiva con una muestra de 47 sujetos, divididos según su relación esqueletal, Clase I= 25, Clase II = 15 y Clase III =7. Resultados: Para la angulación del plano oclusal al inicio y final del tratamiento, utilizando t de student, se encontró diferencia significativa en clase I y II al inicio y al final, la clase III no presentó diferencias. En el plano mandibular utilizando t de student, no se presentaron diferencias en ninguno de los grupos. La correlación de Pearson de PO-PM/SN, encontró correlación en las radiografías iniciales de clase I, II y III y en los casos clase I al final del tratamiento. Para analizar la relación en los casos con o sin extracciones, se utilizó un ANOVA factorial, donde no se encontraron diferencias estadísticamente significativas en ninguno de los grupos. Conclusión: El tratamiento con enfoque anteroposterior, como los realizados en este estudio, produce cambios a nivel oclusal en las relaciones clase I y II.

\section{ABSTRACT}

The inclination of the occlusal plane determines the mandibular position, growth tendency and the dentoskeletal frame. The occlusal plane inclinatión, backwards and upwards, it is seen on class II, on class III, the angle is flatter, promoting a mandibular forward movement. The vertical treatment approach can promote a jaw movement to correct dentoskeletal problems. Objective: To analyze the differences that are manifested in the occlusal plane and mandibular plane at the beginning and the end of orthodontic treatment and their correlation, according to the skeletal frame. Method: retrospective analytical investigation, with a sample of 47 subjects, divided according to their skeletal frame, Class I = 25, Class II $=15$ and Class III $=7$. Results: In the angulation of the occlusal plane at the beginning and end, a significant difference was found in class I and II, the class III did not present significant difference. For the angle of the mandibular plane, there were no statistically significant differences in any of the groups. Pearson's correlation of PO-PM / SN was only found in the initial radiographs in the three skeletal frams and in class I at the end of treatment. In the factorial ANOVA to analyze the relationship in the cases with or without extractions, no statistically significant differences were found in any of the cases. Conclusion: Treatment with an anteroposterior approach, such as those carried out in the study, make changes at the occlusal level in class I and II. 
PALABRAS CLAVE

Plano oclusal, plano mandibular, rotación mandibular, tratamiento vertical, maloclusión

\section{INTRODUCCIÓN}

La diversidad de morfología craneofacial promueve el desarrollo de diferentes maloclusiones y estas han sido tratadas por diferentes corrientes diagnósticas y filosofías de tratamiento. A principios del siglo XX las discusiones diagnósticas y tratamientos, se enfocaban en el plano anteroposterior, sin embargo, se ha evidenciado que una de las mayores dificultades durante el tratamiento de ortodoncia es, el control vertical.2

Dentro de las corrientes que se enfocan en el control vertical, autores como Kim, Sato y Tanaka 2008, en las conclusiones de sus estudios encuentran que, existe una relación entre la inclinación del plano oclusal maxilar posterior con la posición mandibular, que es consistente con la etiología de la clasificación esqueletal del paciente, siendo este plano más inclinado en pacientes clase II y más plano en pacientes clase III..1,2,4,5,6,7. La posición vertical de los dientes posterosuperiores promueve una adaptación funcional de la mandíbula, determinando la angulación mandibular, lo que ayuda a determinar el tipo de crecimiento del paciente, afecta la oclusión funcional e interfiere con el movimiento y posición mandibular.1,2.

\section{Plano oclusal relacionado con el plano mandibular}

El plano Oclusal (PO) es el que identifica el contacto de las caras oclusales de los dientes de ambas arcadas, superior e inferior. 16 Braun y Legan creían en que la oclusión dental estaba influenciada por la posición del

\section{KEYWORDS}

Occlusal plane, mandibular plane, mandibular rotation, vertical treatment, maloclussion

plano oclusal y en 1997 buscaron definir matemática y geométricamente cual era la relación entre la oclusión dental y la rotación del plano oclusal desde una vista sagital, determinaron que aproximadamente existe $0.5 \mathrm{~mm}$ de cambio en la relación oclusal dental por cada grado de rotación del plano oclusal, en cualquier dirección ya sea hacia abajo y hacia atrás o hacia arriba y hacia adelante.14,19.

La propuesta de algunos estudiosos como Kim 1978, Sato y Tanaka 2008, Londoño 2014 entre otros, es corregir los problemas verticales dentales cambiando la inclinación del plano oclusal posterior y con esto reposicionar la mandíbula, para conseguir una resolución que se mantenga estable y funcional.7 La morfología y crecimiento del tercio inferior de la cara está influenciada y guiada por la función de la dentición, donde el crecimiento esqueletal facial y la articulación temporomandibular se ajustan a la posición mandibular.6 El plano oclusal tiene efecto en las funciones orales, oclusión dental, relación de los maxilares y estética facial, criterios importantes para el diagnóstico y finalización del tratamiento. 7

La relación del plano oclusal con la oclusión dental se puede interpretar, en una paciente clase II, existe falta de soporte dental posterior y un plano empinado, con el manejo de planos se puede promover un cambio rotacional antihorario del plano oclusal, haciéndolo más plano, lo cual promueve la rotación mandibular hacia delante, posicionándola anteriormente y mejorando las relaciones dentales.8.13,14,19. 
En un paciente con una relación clase III se encuentra una dimensión vertical aumentada en el sector posterior, con hiperextrusión de molares superiores y un plano oclusal plano, es decir, se encuentra hacia adelante y abajo, sin mucha inclinación.16 El movimiento correctivo mandibular hacia atrás se deriva de una disminución de la altura vertical de los dientes posteriores, ya que fisiológicamente implica una pérdida del soporte dental posterior, y en consecuencia la mandíbula, para compensar la pérdida de soporte oclusal, cambia de posición hacia atrás, adaptándose a la nueva dimensión vertical posterior.7,19. Bassetti (2018) sugiere, corregir con un aumento de la inclinación del plano oclusal, para favorecer la rotación posterior mandibular.16

Lo que indica pequeños cambios en la angulación pueden resultar en cambios significativos en la oclusión, posición mandibular y dental, alcanzando los objetivos en las relaciones dentales del maxilar y la mandíbula.14 Celar 2018, indica que, según el grado de inclinación, se puede determinar la extensión de una maloclusión a nivel anteroposterior. 8 Los sujetos a estudiar estuvieron bajo un diagnóstico y tratamiento de enfoque principalmente anteroposterior, donde el manejo de planos y la rotación mandibular no era prioritaria en la mecánica de tratamiento u objetivos por tanto, lo que se pretende estudiar son los cambios que ocurren con este enfoque de tratamiento, considerando que todos los cambios ortodóncicos influyen en la posición del plano oclusal.

\section{MATERIALES Y MÉTODOS}

Se realizó un estudio analítico retrospectivo, con una población de 128 sujetos, compuesta por los expedientes y radiografías cefalométricas, de pacientes tratados ortodóncicamente en el programa de maestría de ortodoncia y ortopedia maxilofacial de la Universidad de San Carlos de Guatemala, de inicio a fin y presentados como casos "board de excelencia académica" durante los años 2014-2018. La muestra se calculó con la siguiente fórmula: $n=\frac{\mathrm{NZ}^{\wedge} \mathrm{pq}}{\mathrm{e}^{\wedge}(\mathrm{N}-1)+\left(\mathrm{Z}^{\wedge} \mathrm{pq}\right)}$ tomando la población (Z) de 128 sujetos, un nivel de significancia de $95 \%$ (p) con un margen de error del $0.05(q)$, resultando en una muestra de 47 sujetos.

Para comprender mejor el comportamiento de los planos, se tomó el porcentaje de cada una de las relaciones esqueletales de la población y se proyectó en la muestra, distribuyendo los grupos de la siguiente manera: Clase I $53.19 \%$ (25), clase II $31.91 \%$ (15) y clase III 14.89 (7). Los casos se seleccionaron aleatoriamente con la función "azar" de Excel, para obtener los sujetos que componen de la muestra. La muestra se seleccionó según los criterios de inclusión y exclusión, posterior a la autorización del comité de bioética de la Universidad de San Carlos.

Se recolectaron datos como, número de registro, ausencia o presencia de extracciones de premolares durante el tratamiento, ángulos y medidas lineales que se tomaron en cuenta en el estudio. Estas medidas se tomaron para establecer las relaciones oclusales y esqueléticas, así como la inclinación del plano oclusal.

Las radiografías de inicio y final en cada uno de los sujetos, fueron trazadas por la autora del estudio. Los trazados se elaboraron manualmente con papel vegetal, lápiz y lapiceros de colores, donde se localizaron las medidas necesarias para el estudio.

Para establecer la relación esqueletal, se trazó la radiografía de inicio del tratamiento, obteniendo los resultados de las siguientes medidas cefalométricas. 
Ángulos: SNA, SNB, ANB, convexidad facial, profundidad maxilar y APDI.

Posteriormente se tomaron las medidas de plano oclusal y plano mandibular, necesarias para realizar el estudio.

Prueba de plano oclusal los ángulos que se analizaron fueron: plano oclusal a SN, inclinación del plano oclusal según $\mathrm{Xi}$, y plano oclusal con eje mandibular.
Para evaluar el plano mandibular los ángulos utilizados fueron plano mandibular (Go-Gn) a SN, nasion perpendicular - Pogonion y ODI. En la correlación de Pearson se analizaron los ángulos de plano oclusal/sella nasion y plano mandibular/sella nasion.

Y por último para el ANOVA factorial se analizaron los ángulos de plano oclusal a SN, inclinación del plano oclusal según Xi, y plano oclusal con eje mandibular.

Figura 1. Plano Frankfort (FH)

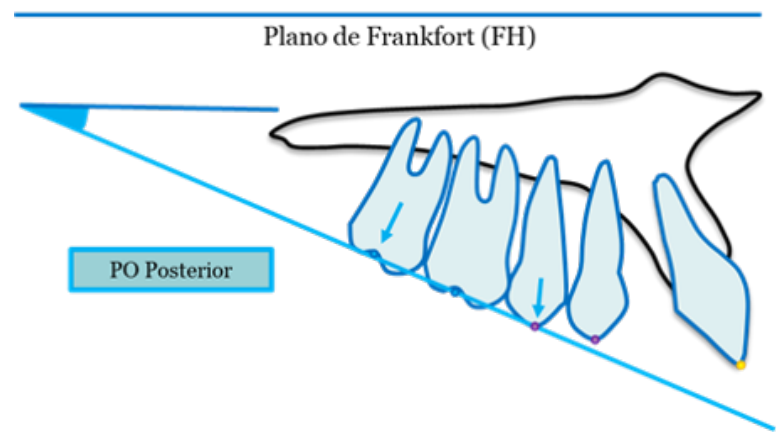

\section{RESULTADOS}

Análisis plano oclusal antes y después del tratamiento según su relación esqueletal.

Prueba T de variables relacionadas para plano oclusal al inicio y final del tratamiento.
En cada una de las pruebas se tomó un nivel de significancia inferior a al 0.05 , para aceptar la hipótesis de investigación, si el nivel de significancia es mayor a 0.05 se acepta la hipótesis nula indicando que no existen diferencias estadísticamente significativas.

Tabla 1. Prueba T de variables relacionadas Inicio-Final

\begin{tabular}{|c|c|c|c|}
\cline { 2 - 4 } \multicolumn{1}{c|}{} & Clase I & Clase II & Clase III \\
\hline PO-SN & 0.001 & 0.023 & 0.495 \\
\hline Altura PO-Xi & 0.288 & 0.027 & 0.52 \\
\hline PO-Eje Md & 0.02 & 0.018 & 0.941 \\
\hline
\end{tabular}


En estos casos el nivel de significancia para rechazar la hipótesis nula y aceptar la hipótesis de investigación aceptando que existe una diferencia estadísticamente significativa al inicio y al final de tratamiento únicamente se afirma para la relación esqueletal clase I en los ángulos PO-SN y PO-Eje Md, y en todas las medidas tomadas para clase II, en las otras mediciones y relación esqueletal clase III, se acepta la hipótesis nula indicando que no existe diferencia estadísticamente significativa entre los planos en cuestión.

Análisis plano mandibular antes y después del tratamiento según su relación esqueletal. Prueba T de variables relacionadas para plano mandibular al inicio y final del tratamiento.

Tabla 2. Prueba $T$ de variables relacionadas Inicio-Final

\begin{tabular}{|l|c|c|c|}
\cline { 2 - 4 } \multicolumn{1}{c|}{} & Clase I & Clase II & Clase III \\
\hline SN-PM & 0.007 & 0.834 & 0.857 \\
\hline Na perpendicular-Pog & 0.314 & 0.283 & 0.244 \\
\hline ODI & 0.735 & 0.8 & 0.122 \\
\hline
\end{tabular}

En estos casos el nivel de significancia para rechazar la hipótesis nula y aceptar la hipótesis de investigación aceptando que existe una diferencia estadísticamente significativa al inicio y al final de tratamiento únicamente se afirma para la relación esqueletal clase I en el ánglulo SN-PM, en el resto de ángulos estudiados en clase I y para las relaciones esqueletales clase II y III, se acepta la hipótesis nula indicando que no existe diferencia estadísticamente significativa entre los planos en cuestión.

Análisis del plano oclusal y el plano mandibular antes y después del tratamiento de ortodoncia, según su clasificación esqueletal.

Correlación de Pearson, Plano Oclusal y Plano Mandibular según SN.

En cada uno de los casos se realizó una gráfica de puntos para determinar el comportamiento de la correlación entre las variables, en cada una de las mediciones estudiadas se encuentra una correlación de variables positiva, donde si el ángulo del plano oclusal aumentaba el del plano mandibular también aumentaba, de igual forma si disminuía, por lo tanto, se consideraron todos los casos con correlación positiva como muestra la gráfica del ejemplo a continuación: 
Figura 2. Ángulo PC-SN Final

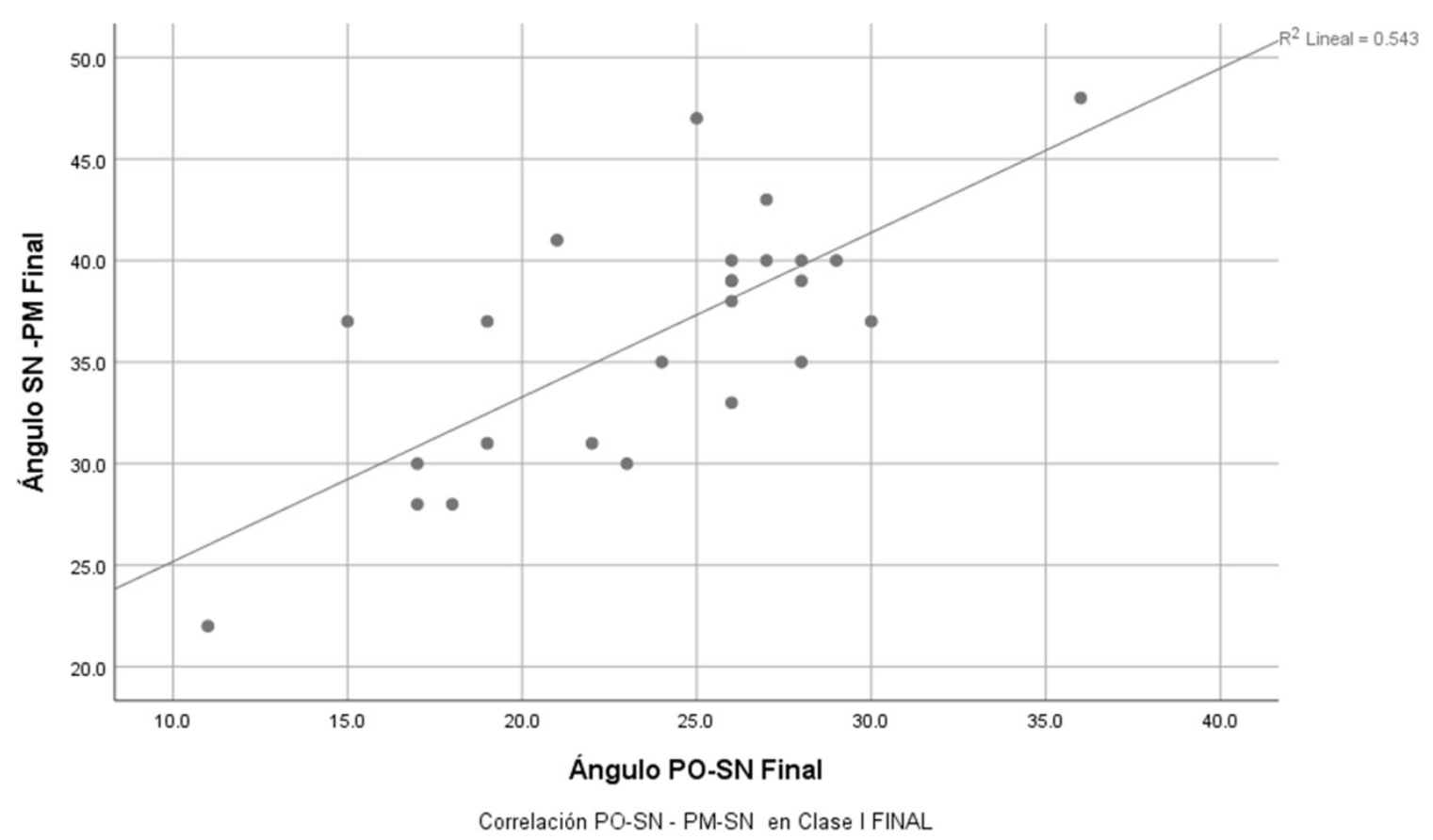

Para este análisis se puede afirmar que existe una correlación entre las variables si el resultado se encuentra entre 0.7 y 0.9 , en este caso se puede afirmar que existe una correlación entre ambos planos, con un con un nivel de confiabilidad del 95\%, por consiguiente se rechaza la hipótesis nula y se acepta la hipótesis alterna.

Tabla 3. Correlación de Pearson Po-Pm según SN

\begin{tabular}{|l|c|c|}
\cline { 2 - 3 } \multicolumn{1}{c|}{} & \multicolumn{2}{c|}{ Po-Pm según SN } \\
\cline { 2 - 3 } \multicolumn{1}{c|}{} & Inicio & Final \\
\hline Clase I & 0.74 & 0.737 \\
\hline Clase II & 0.852 & 0.611 \\
\hline Clase III & 0.794 & 0.168 \\
\hline
\end{tabular}

En este estudio se puede observar que sí existe una correlación en el plano oclusal y el plano mandibular al inicio del tratamiento, sin embargo únicamente hay correlación al final del tratamiento en los casos clase I, los clase II y III no tienen ninguna correlación significativa al final del tratamiento.
Análisis plano oclusal en relación con, la ausencia o presencia de extracciones según su relación esqueletal

En este caso los sujetos se dividieron, según la presencia o ausencia de extraciones por lo cual el grupo se dividió de la siguiente manera: 
Tabla 4.

\begin{tabular}{|l|c|c|}
\hline \multirow{2}{*}{ Clase I } & Con Extracciones & 12 \\
\cline { 2 - 3 } & Sin Extracciones & 13 \\
\hline \multirow{2}{*}{ Clase II } & Con Extracciones & 10 \\
\cline { 2 - 3 } & Sin Extracciones & 5 \\
\hline \multirow{2}{*}{ Clase III } & Con Extracciones & 4 \\
\cline { 2 - 3 } & Sin Extracciones & 3 \\
\hline
\end{tabular}

Para determinar si existe diferencia significativa entre los grupos, el nivel de significancia debe ser inferior a 0.05 , en este caso se rechaza la hipótesis nula y se acepta la hipótesis de investigación que afirma que sí existen diferencias estadísticamente significativas entre los grupos.

Cuadro resumen, análisis plano oclusal en relación con, la ausencia o presencia de extracciones según su relación esqueletal

Tabla 5. Nivel de significancia entre

\begin{tabular}{|l|c|c|c|c|c|c|}
\cline { 2 - 7 } \multicolumn{1}{c|}{} & \multicolumn{2}{c|}{ Extracciones } & \multicolumn{2}{c|}{ Relación esqueletal } & \multicolumn{2}{c|}{ Interacción } \\
\cline { 2 - 7 } \multicolumn{1}{c|}{} & $\mathrm{F}$ & $\mathrm{P}$ & $\mathrm{F}$ & $\mathrm{P}$ & $\mathrm{F}$ & $\mathrm{P}$ \\
\hline PO-SN Inicio & 0.638 & 0.429 & 1.701 & 1.95 & 0.78 & 0.465 \\
\hline PO-SN Final & 0.023 & 0.881 & 1.43 & 0.251 & 0.607 & 0.55 \\
\hline Altura PO-Xi Inicio & 0.147 & 0.703 & 0.868 & 0.427 & 0.457 & 0.636 \\
\hline Altura PO-Xi Final & 0.025 & 0.876 & 0.643 & 0.531 & 0.172 & 0.843 \\
\hline PO-Eje Md Inicio & 0.399 & 0.531 & 0.213 & 0.809 & 0.995 & 0.378 \\
\hline PO-Eje Md Final & 2.087 & 0.156 & 2.138 & 0.131 & 0.939 & 0.399 \\
\hline
\end{tabular}

En cada una de las mediciones realizadas, el nivel de significancia es mayor a 0.05 , por lo que se puede afirmar que se acepta la hipótesis nula y se rechaza la hipótesis de investigación, concluyendo que no existen diferencias estadísticamente significativas al inicio y al final del tratamiento en los casos con o sin extracciones según su relación esqueletal.

\section{ANÁLISIS}

Ya establecida la relación esqueletal de cada uno de los sujetos y seleccionada la muestra, los datos se almacenaron en una hoja de
Excel, para luego ser trasladados al programa SPSS donde en cada una de las mediciones se realizaron los análisis de estadísticos descriptivos, también se obtuvieron datos de normalidad, con las pruebas de Shapiro Wilk y Kolmogorov-Smirnov, datos de homocedasticidad, con la prueba de Levin.

Para obtener los resultados del estudio se realizaron 3 pruebas estadísticas, en el programa SPSS donde se encuentra la prueba $\mathrm{T}$, diferencias de medias del plano oclusal y mandibular al inicio y final del tratamiento, Correlación de Pearson, la correlación entre el plano oclusal y mandibular y ANOVA factorial, plano oclusal con ausencia o presen- 
cia de extracciones, en todos los casos los análisis se realizaron dividiendo a los sujetos según su relación esqueletal.

\section{DISCUSIÓN}

Entre los objetivos del estudio se buscaba determinar si existen diferencias en las angulaciones de los planos oclusal y mandibular, con el fin de evaluar otras alternativas de tratamiento con enfoque vertical para diagnosticar y tratar a los pacientes que se presentan al programa de maestría.

Baca et al (2008) sugieren que "Debido a que la mecánica ortodóncica, sólo puede intervenir en la modificación del complejo dentoalveolar, enfocar los tratamientos hacia el manejo del plano oclusal posterior ayudaría a mejorar la relación de los maxilares", en este caso Baca et al se refiriere a la relación esqueletal clase II.19 Es esta una de las razones por las cuales, el plano oclusal posterosuperior, es analizado en este estudio, donde se encontró en los ángulos del plano oclusal con sella-nasion y el plano mandibular, comportamientos similares, donde para clase I en ambos ángulos sí existe diferencia estadísticamente significativa entre los grupos al inicio y al final de tratamiento, por lo cual, se pude afirmar que existen cambios en la angulación del plano oclusal durante el tratamiento, sin embargo, no existe diferencia significativa en la posición este, con respecto al punto medio de la rama mandibular (Xi).

Los resultados para clase II muestran un comportamiento similar en cada una de las medias, donde existe diferencia estadísticamente significativa en los ángulos de sella-nasion y Eje mandibular, con el plano oclusal, así como la posición de este, en relación con $\mathrm{Xi}$, por el contrario, en relación con las mediciones realizadas en clase III no existe diferencia estadísticamente significa- tiva en ninguno de los ángulos o medida anteriormente mencionadas.

Respecto a la posición mandibular, Giraldo et al (2012), afirma que "La altura vertical de la oclusión puede afectar la inclinación del plano oclusal posterior y subsecuentemente la posición y función mandibular en diferentes tipos de maloclusiones esqueléticas". 22 El movimiento que se realiza en el plano oclusal posterior afecta la posición final de la mandíbula, en base a esto según el análisis de $t$ de student para el plano mandibular, se observa diferencia estadísticamente significativa al inicio y final del tratamiento únicamente en el ángulo sella-nasion/plano mandibular, de los casos clase I, en las demás medidas, para la relación esqueletal clase I y todas las mediciones en los grupos de clase II y III, no se encontró diferencia estadísticamente significativa antes y después de realizado el tratamiento de ortodoncia.

Autores como Tanaka, E. and Sato, S (2008) y Giraldo et al (2012), afirman que: la inclinación del plano oclusal es uno de los factores de la adaptación dentoalveolar, que influyen en los cambios anteroposteriores, dentales y mandibulares.6.22. La rotación del plano oclusal, en sentido antihorario, induce a un movimiento mesial del molar inferior. Cada grado de rotación del plano oclusal puede resultar en $0.5 \mathrm{~mm}$ de cambio en la relación molar.22 Es por esto que, el presente estudio no encuentra cambios a nivel mandibular a pesar de encontrar cambios a nivel oclusal en la evaluación inicial y final del tratamiento, para que se produzcan cambios significativos en la posición mandibular y por consecuencia en las relaciones molares y esqueletales los movimientos a nivel oclusal deben ser más pronunciados que los que se encuentran en este estudio.

Los estudios realizados por Fukoshima et al (1996) Akimoto et al (2009), Tanaka et al 
(2008), entre otros, muestra la importancia que tiene el plano oclusal, para el desarrollo de los maxilares y la posición final de la mandíbula, la cual se adapta a la angulación que el plano oclusal superior le proporciona.1.6,16 Los resultados muestran correlación entre el plano oclusal y el plano mandibular, en los ángulos con sella-nasion, cada una de las medidas son estadísticamente significativas al inicio y al final del tratamiento en los casos de clase I, sin embargo, en las relaciones clase II y III es estadísticamente significativa únicamente al inicio del tratamiento, no al final de este. En todos los casos, existe una correlación de variables positiva es decir al aumentar el ángulo en el plano oclusal, aumenta el ángulo en el plano mandibular y viceversa. Tal como lo describe Bassetti (2018) la mandíbula sufre un proceso de adaptación al plano oclusal, donde al tener una posición hacia abajo, un ángulo menor, la mandíbula realiza un movimiento antihorario, girando hacia arriba y a adelante disminuyendo la inclinación del plano, tal como se demuestra la correlación de todas las relaciones esqueletales de este estudio al inicio del tratamiento.16 Giraldo (2012) resalta en sus resultados, consistencia en el comportamiento del plano oclusal según la relación esqueletal, encontrando en los clase I un plano oclusal estable, inclinado para clase II y plano para clase III tal como se muestran en este estudio. 22

Yamaguchi y Nanda (1991) y Baca et al (2008) estudiaron las diferencias existentes entre los tratamientos con o sin extracciones, principalmente en la clase II.19,26. Yamaguchi y Nanda (1991) encuentra diferencias en la rotación del plano mandibular en los casos con extracciones, por el contrario, los casos sin extracciones no presentan ningún efecto significativo dental o esqueletal.26. Por el contrario, Baca et al (2008) así como en este estudio, no encontraron diferencias estadís- ticamente significativas entre los grupos con y sin extracciones.19 El Anova factorial, que se realizó a los datos del presente estudio muestran que el plano oclusal al inicio y final del tratamiento, en cada una de las relaciones esqueletales, y medidas evaluadas, en presencia o ausencia de extracciones, no tiene diferencias estadísticamente significativas entre los grupos.

\section{CONCLUSIONES}

Existen modificaciones en la inclinación del plano oclusal al inicio y al final del tratamiento, en la relación clase I y clase II, por el contrario, en los casos de relación esqueletal clase III no se observan cambios significativos a nivel oclusal.

El tratamiento con enfoque anteroposterior o sagital, como el que se realizaron los tratamientos de este estudio, produce cambios a nivel oclusal en las relaciones clase I y II, sin embargo, no son significativos a nivel mandibular.

No hay diferencias significativas en la angulación del plano mandibular, ni en la posición mandibular antes y después del tratamiento en las relaciones esqueletales clase II y III. Considerando la influencia de la inclinación del plano oclusal en la posición mandibular, la modificación en el plano oclusal debe ser mayor para obtener movimientos a nivel mandibular.

Existe una correlación positiva en la posición del plano oclusal con la posición del plano mandibular en las tres relaciones esqueletales al inicio del tratamiento, donde al aumentar el ángulo en el plano oclusal aumenta en el plano mandibular, al finalizar el tratamiento la correlación entre los planos oclusales y mandibulares es significati- 
va únicamente en clases I. No se encuentra correlación al final del tratamiento para la relación esqueletal clase II y III.

No se encontró diferencia estadísticamente significativa antes y después del tratamiento de ortodoncia en el comportamiento del plano oclusal, de los casos con o sin extracciones independientemente de su relación esqueletal.

\section{PERSPECTIVAS DE FUTURO}

- Dentro de los programas de maestría, se debería reforzar los diagnósticos y tratamiento con enfoque vertical, así como el análisis de los planos oclusales, ante, para influir de forma diferente y complementaria en los tratamientos ortodóncicos.

- Estudios longitudinales más grandes y extensos donde se evalúen y midan cambios en la angulación oclusal y el movimiento mandibular resultante.

- Realizar una investigación extensa, sobre el comportamiento de los planos, para la relación esqueletal clase III, debido a la cantidad de casos clase III de este estudio.

- Ampliar un estudio del comportamiento de los maxilares antes y después del tratamiento en una clasificación de crecimiento hiper o hipodivergente, donde se comparen los casos con y sin extracciones.

\section{REFERENCIAS}

Akimoto, S. Kubota, M. and Sato, S. (2010) Increase in vertical dimensión and maxilo-mandibular growth in a longitudinal growth sample. Kanagawa: Japón. J. Stomat. Occ. Med. (2010) 3:15-19. https://doi.org/10.1007/s12548-0100040-x

Arriola-Guillén, Land Flores-Mir, C. (2014) Molar heights and incisor inclinations in adults with Class II and Class III skeletal open-bite malocclusions. Am J Orthod Dentofacial Orthop 2014;145:325-32. https:// doi.org/10.1016/j.ajodo.2013.12.001

Bassetti, N. (2018) La dimensión vertical en Prótesis y Ortognatodoncia: Integración entre función y estética. Amolca: Venezuela pp 206

Bench, R W., Hilgers, J.J.: Bio-progressive therapy, J. Clini. Orthodont.1977 11:820-843.

Björk, A. (1969) Prediction of mandibular growth rotation. Copenhagen: Dinamarca. Am. J. Orthodontics. June 1969. Vol. 55. No. 6. 585-599. https://doi.org/10.1016/00029416(69)90036-0

Braun, S. and Legan, H (1997) Changes in occlusion related to the cant of the occlusal plane. Am J Orthod Dentofac Orthop. 1997;111:184-8. https://doi. org/10.1016/S0889-5406(97)70214-2

Celar, A. et al (2018) Association of anterior and posterior occlusal planes with different Angle and skeletal classes in permanent dentitions. J Orofac Orthop (2018) 79:267-276. https://doi. org/10.1007/s00056-018-0139-z

Fushima, K. et al. (1996) Significance of the cant of occlusal plane in class II division 1 malocclusion. Eur J. Orthodont 1996; 18:2740. https://doi.org/10.1093/ejo/18.1.27

Giraldo, A. et al. (2012) Caracterización del plano oclusal y diferentes marcos dentoesqueléticos en escolares entre 5 y 6 años. Rev. CES Odont. 2012;25(1) 32-41 Gregoret, J. et al. (1997) Ortodoncia y cirugía ortognática, diagnóstico y planificación. España: ESPAXS pp 135-173 
Jarabak J R Fizzel J A (1972) Technique and treatment with lightwire edwise appliances, 2nd. The C.V. Mosby Company, Saint Louis.

Kenji Fukushima (1996) Significance of the cant of the posterior occlusal plane in class II division 1 malocclusions. European Journal of Orthodontics (1996) 18: 27-40. https://doi.org/10.1093/ ejo/18.1.27

Kim, Y. and Vietas, J. (1978) Anteroposterior dysplasia indicator: An adjunct to cephalometric differential diagnosis. Am J. Orthod June 1978 vol. 73 No. 6. https://doi.org/10.1016/00029416(78)90223-3

Kim Y (1974) Overbite Depth indicator with particular reference to anterior open-bite Am J. Orthod june 1974 Vol. 65 No 6. https: / /doi.org/10.1016/00029416(74)90255-3

Kim et al (2002) Transition of molar relationships in different skeletal growth patterns. Am J Orthod Dentofacial Orthop 2002;121:280-90. https://doi. org $/ 10.1067 / \bmod .2002 .119978$

Kim e Yong et al, Transition of molar relationships in different skeletal growth patterns. Am J Orthod Dentofacial Orthop. 2002;121:280-90.

Proffit, W. et al (2014) Ortodoncia contemporanea España: Elsevier Ed. 5

Reyes, I. Gonzalez, T. and Londoño, L. (2014) Modificación del plano oclusal y la maloclusión esquelética en radiografías de perfil de pacientes con tratamiento de ortopedia miofuncional. Bogotá, Colombia. Revista Cient. Soc. Colomb. Ortod. 2014; 1(1): 37-44.

Sadao Sato. Alteration of occlusal plane due to posterior discrepancy relates to development of malocclusion - Introduc- tion to Denture Frame Analysis. Bull of Kanagawa Dental College. 198715 (2): 115-123.

Sato,S. Akimoto, S, et al (2001) Orthodontic Therapy Using Multiloop Edgewise ArchWire. Manual of the clinical application of MEAW Technique. Cap 4 Pp 23-28.

Baca, D. et al (2008) Comparación de la variación del plano oclusal con la posición mandibular en tratamientos clase II división 1 con y sin exodoncias. CIELO: 19-29.

Silva. R. (2009) Young H. Kim, Cephalometric Analytic Procedure. Departent Of Orthodontics, Latin-American University, ULA, México. p.1-12 (On Line). Disponible en: https://pdfs. semanticscholar.org/5198/9fb59dfc12c9333348e137c4ccdb4546de7a.pdf?

Tanaka, E. and Sato, S (2008) Longitudinal alteration of the occlusal plane and development of different dentoskeletal frames during growth, Yokosuka, Japan, Am J Orthod Dentofacial Orthop 2008; 134:602 e1-602.e1. https://doi. org/10.1016/j.ajodo.2008.02.017 1

Ugalde, F (2007) Clasificación de la maloclusión en los planos anteroposterior, vertical y transversal. Revista ADM 2007; LXIV (3):97-109

Yamaguchi, K y Nanda, R. (1991) The effects of extraction and nonextraction treatment on the mandibular position. Am J Orthod Dentofac Orthop 1991;100:443-52. https://doi.org/10.1016/0889-5406(91)70084-A

Zamora, C (2014) Compendio de Cefalometría - Análisis clínico práctico. Colombia: Amolca. 


\title{
SOBRE AUTORES
}

\section{Dra. Vilma Isabel Jofre Ortiz}

\author{
Es Cirujana Dentista, Maestría en Ortodoncia \\ y Ortopedia Maxilofacial.
}

\section{Dr. Bienvenido Argueta Hernández}

\section{Es Doctor en Educación y Estudios Cultura- les de la Universidad de Ohio. Investigador social y políticas públicas en la Universidad de San Carlos de Guatemala y profesor en metodología de investigación en la Facultad de Odontología de la USAC.}

\section{Conflicto de intereses}

Declara no tener ningún conflicto de intereses.

Declaración de consentimiento informado

El estudio se realizó, respetando el Código de ética y buenas prácticas editoriales de publicación.

\section{Derechos de uso}

Copyright@ 2021 por Vilma Isabel Jofre Ortiz, Bienvenido Argueta Hernández y Gerson Rudick Chinchilla Dubón Este texto está protegido por una licencia Creative Commons 4.0. Internacional

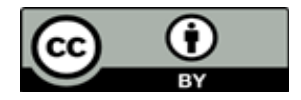

Usted es libre para compartir, copiar y redistribuir el material en cualquier medio o formato y adaptar el documento, remezclar, transformar y crear a partir del material para cualquier propósito, incluso comercialmente, siempre que cumpla la condición de atribución: usted debe reconocer el crédito de una obra de manera adecuada, proporcionar un enlace a la licencia, e indicar si se han realizado cambios. Puede hacerlo en cualquier forma razonable, pero no de forma tal que sugiera que tiene el apoyo del licenciante o lo recibe por el uso que hace. 